\title{
Neural response to emotional faces with and without awareness: event-related fMRI in a parietal patient with visual extinction and spatial neglect
}

\author{
P. Vuilleumier ${ }^{\text {a,* }}$, J.L. Armony ${ }^{\mathrm{a}, \mathrm{b}}$, K. Clarke ${ }^{\mathrm{a}}$, M. Husain ${ }^{\mathrm{a}, \mathrm{c}}$, J. Driver ${ }^{\mathrm{a}}$, R.J. Dolan ${ }^{\mathrm{b}, \mathrm{d}}$ \\ ${ }^{a}$ Institute of Cognitive Neuroscience, University College London, Alexander House, 17 Queen Square, London WC1N 3AR, UK \\ ${ }^{\mathrm{b}}$ Wellcome Department of Cognitive Neurology, Functional Imaging Laboratory, London, UK \\ ${ }^{\mathrm{c}}$ Imperial College School of Medicine, London, UK \\ d Royal Free Hospital, University College School of Medicine, London, UK
}

Received 2 November 2001; received in revised form 8 March 2002; accepted 15 March 2002

\begin{abstract}
This study examined whether differential neural responses are evoked by emotional stimuli with and without conscious perception, in a patient with visual neglect and extinction. Stimuli were briefly shown in either right, left, or both fields during event-related fMRI. On bilateral trials, either a fearful or neutral left face appeared with a right house, and it could either be extinguished from awareness or perceived. Seen faces in left visual field (LVF) activated primary visual cortex in the damaged right-hemisphere and bilateral fusiform gyri. Extinguished left faces increased activity in striate and extrastriate cortex, compared with right houses only. Critically, fearful faces activated the left amygdala and extrastriate cortex both when seen and when extinguished; as well as bilateral orbitofrontal and intact right superior parietal areas. Comparison of perceived versus extinguished faces revealed no difference in amygdala for fearful faces. Conscious perception increased activity in fusiform, parietal and prefrontal areas of the left-hemisphere, irrespective of emotional expression; while a differential emotional response to fearful faces occurring specifically with awareness was found in bilateral parietal, temporal, and frontal areas. These results demonstrate that amygdala and orbitofrontal cortex can be activated by emotional stimuli even without awareness after parietal damage; and that substantial unconscious residual processing can occur within spared brain areas well beyond visual cortex, despite neglect and extinction. (C) 2002 Elsevier Science Ltd. All rights reserved.
\end{abstract}

Keywords: Visual neglect; Visual extinction; Emotion; Faces; Amygdala; Awareness

\section{Introduction}

Fast detection of potential threats is important for many organisms. It has been suggested that the amygdala may constitute a dedicated system for processing danger and emotionally-relevant events, especially when these occur outside attention or awareness [4,30,31]. Studies of fear-conditioning in animals suggest that the amygdala may receive direct thalamic inputs bypassing striate and extrastriate visual cortex [4,31]. Functional imaging studies in humans reported amygdala activation by emotional stimuli, such as fearful or fear-conditioned faces, even when masked and hence not detected by healthy subjects $[36,37,51]$. Similarly, the amygdala can still be activated by fearful faces when these are task-irrelevant and outside the focus of

\footnotetext{
* Corresponding author. Tel.: +44-20-7679-1177; fax: +44-20-7813-2835

E-mail address: p.vuilleumier@ucl.ac.uk (P. Vuilleumier).
}

attention [46]. Moreover, behavioural findings indicate that normal observers may exhibit automatic responses (such as discriminative skin conductance changes) to emotional stimuli that are neither overtly attended nor consciously detected (e.g. [17]).

In the present study, we used event-related fMRI in a patient with hemispatial neglect and visual extinction, to investigate the neural responses evoked by emotional faces with and without conscious perception. Neglect often follows right inferior parietal damage, and is characterised by impaired attention and lack of awareness for stimuli on the contralesional (left) side of space [24]. Such patients may have intact visual fields but show perceptual extinction, i.e. they may fail to detect a stimulus in the contralesional field when it is presented with a concurrent stimulus in the ipsilesional field, although the same contralesional stimulus can be detected when presented alone $[7,13,42]$. In these patients, visual pathways into occipital and temporal cortex may be anatomically spared, 
including the amygdala. Behavioural findings and recent functional imaging studies suggest that these intact visual pathways can still sustain some residual "implicit" processing of extinguished stimuli, despite the patients' unawareness $[8,43,47]$. Likewise, emotional significance of stimuli may be detected despite contralesional inattention on bilateral simultaneous events. Extinction can be less for faces with an emotional than neutral expression [48], or for threat-related stimuli, such as spiders than neutral stimuli, such as flowers [49]. A plausible neural substrate for this preserved processing of contralesional emotional stimuli might involve the amygdala, possibly interacting with intact visual areas to prioritise awareness for salient stimuli $[4,30,35]$.

Our study examined the neural correlates of emotional face processing, associated with either awareness or unawareness of the faces, in a patient who had a focal right-parietal lesion with intact visual fields and reliable left visual extinction on bilateral simultaneous stimulation (BSS). Photographs of faces with neutral or fearful expressions were presented either alone in the right visual field (RVF) or left visual field (LVF), or in the LVF together with a concurrent photograph of a house in the RVF (BSS). The critical events were the latter BSS trials, where our patient extinguished a substantial proportion of left-side faces. The major questions were whether extinguished fearful faces would still elicit emotional responses in amygdala and other limbic structures; and whether these responses would differ as a function of awareness versus extinction of the faces.
Our main analysis could therefore employ a $2 \times 2$ factorial design for the critical BSS trials, testing for the main effects of emotion (neutral or fearful face in LVF) irrespective of awareness; as well as for any interaction of such emotional effects with awareness of the faces (versus extinction). In addition, we could also contrast BSS trials with an extinguished LVF face to unilateral trials with a RVF house alone (same conscious percept reported), allowing us to examine any implicit effects due to extinguished stimuli $[43,47]$.

\section{Materials and methods}

\subsection{Patient}

GK is a 69-year-old man, who suffered a right-hemisphere infarction 2 years prior to the current study, centred on posterior inferior parietal region and underlying white matter (Fig. 1). On neurological examination, he still showed a mild loss of dexterity and position-sense in the left hand, reliable left extinction on bilateral simultaneous visual (and tactile) stimulation, and intact visual fields on both sides. He scored 87 on the standardised behavioural inattention test ( $<$ cut-off 129) and still showed moderate left spatial neglect in standard clinical tests, such as Mesulam cancellation task (25 left-side omissions per 60 targets) at the time of investigation. Additional behavioural [25] and imaging [43] data were previously reported.

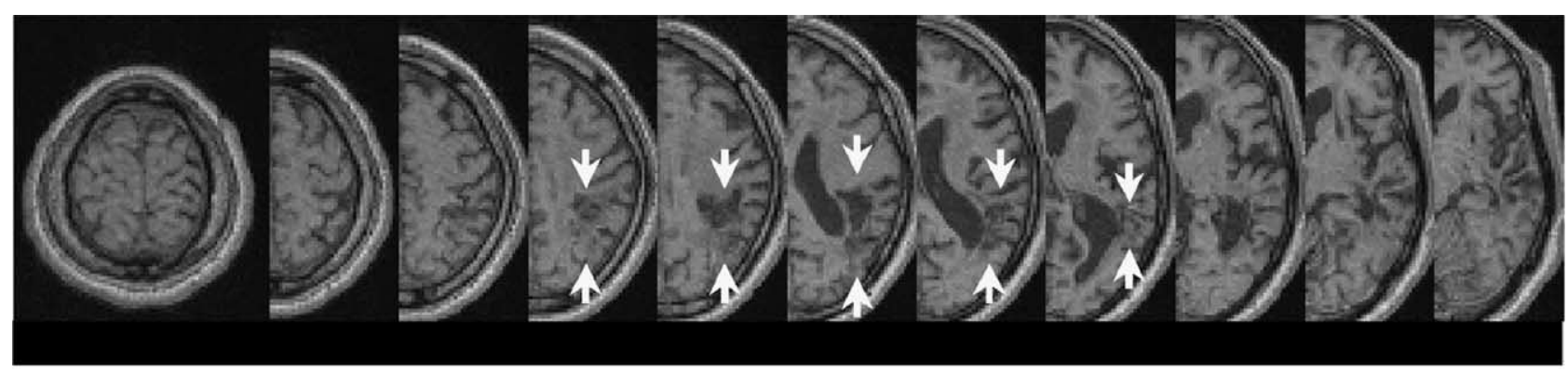

(A)

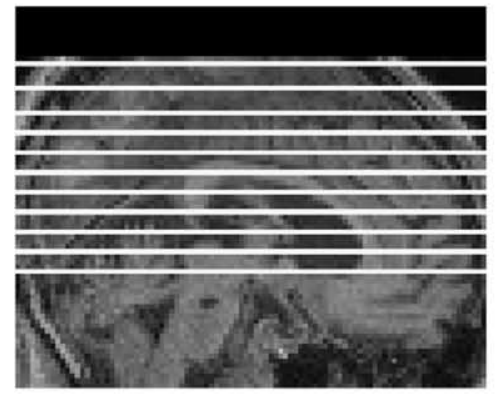

(B)

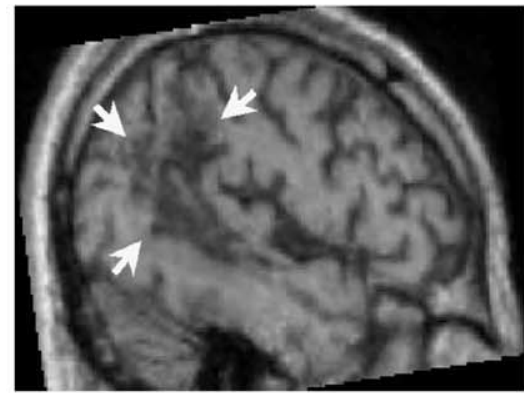

(C)

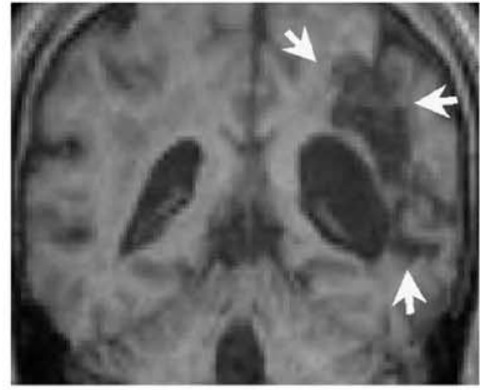

(D)

Fig. 1. Illustration of the patient's brain lesion. T1-weighted structural MRI scan sections show a hypodense area in right inferior parietal cortex, consistent with chronic infarction. Visual areas in occipital and temporal cortex are intact. 


\subsection{Behavioural task outside the scanner}

GK was trained on our extinction-task, a few days before scanning, using different stimuli to those subsequently shown in the scanner. A further training session was given in the scanner just before the actual fMRI session, again using different stimuli. During these sessions, photographs of faces and houses were presented either unilaterally on the left- or right-side, or bilaterally on both sides, with the probabilities of bilateral versus unilateral, left versus right, and face versus house stimuli all equally balanced. Bilateral trials included a face on one side and a house on the other side, with faces equally often in the LVF or RVF. All trial types were intermingled in random order. The patient reported by key-presses via his ipsilesional hand whether he saw a face (thumb response), a house (index), or both stimulus types (middle finger). To ensure central fixation during the task, each trial began with a fixation point at the centre of the screen which turned either green or red $750 \mathrm{~ms}$ before stimulus onset, instructing the patient either to respond normally (green "go" fixation; $2 / 3$ trials) or to withhold from responding (red "no-go" fixation; $1 / 3$ trials). On $15 \%$ of trials (catch), a fixation point was not followed by any stimulus. An error message and a loud warning sound followed incorrect key-presses. During these training sessions, GK failed to detect a face or house in the LVF on $40-100 \%$ of bilateral displays, depending on exposure duration (100-1000 ms in different blocks). His performance during the final training-blocks, inside the scanner, was used to determine stimulus duration for the subsequent scanning sessions $(600 \mathrm{~ms})$, with the aim of obtaining $\sim 50 \%$ of extinction on critical BSS trials, yet with reliable detection on unilateral LVF trials.

\subsection{Experimental task inside the scanner}

Three successive fMRI scanning sessions were performed within the same day ( 9 min each). The task was similar to the previous training, except for a different proportion of trial types and a different set of stimuli. Ten faces (from Ekman and Friesen [16]) and 10 houses were used (Fig. 2), back-projected on a mirror mounted on the head-coil, and appearing briefly $(600 \mathrm{~ms})$ on either side $\left(\sim 8^{\circ}\right.$ away from fixation, $\sim 2^{\circ}$ of visual angle each). All faces and houses were shown within the same black oval frame to avoid discrimination based on outer contours. Response keys were mounted on a custom-made glove attached to the unseen right hand.

The critical events were BSS with a LVF face and a RVF house, where the face had either a neutral or fearful expression (24 trials for each of these conditions per session). Based on training data, we expected that the patient would extinguish the LVF faces on $\sim 50 \%$ of these trials. No BSS trials with a RVF face and a LVF house occurred during actual scanning, in order to minimise the number of trial types, and so optimise our statistical power for the critical experimental comparisons. There were unilateral events with a fearful or neutral face in LVF or RVF (six trials each per session), and unilateral events with a house in LVF or RVF ( 6 and 12 trials per session, respectively). As in training, trials began with a green (go) or red (no-go) fixation point, but the latter now occurred only on some unilateral trials with a left-side house or right-side face ( $9 \%$ of total trial types). All trial types were similarly randomised and distributed in the three scanning sessions, with a mean interval of $5.1 \mathrm{~s}$ (randomly jittered between 4.5 and $13 \mathrm{~s})$.

\section{4. fMRI imaging}

MRI data were acquired on a $2 \mathrm{~T}$ Siemens VISION system with a head volume coil. Functional images were acquired with a gradient echo-planar $\mathrm{T}^{*}$ sequence using BOLD (blood oxygenation level dependency) contrast. A total of 460 functional images were taken during three scanning sessions (32 contiguous axial slices, $3 \mathrm{~mm}$ thickness), in addition to eight dummy images at the beginning of each session that were subsequently discarded, to allow for T1 equilibration effects. TR was $2.93 \mathrm{~s}$ and average inter-stimulus time was $5.1 \mathrm{~s}$. A structural MRI scan was also acquired using a T1-weighted sequence to obtain anatomical images of the patient's brain and lesion.

The fMRI data were analysed using the general linear model for event-related designs in SPM99 (http://fil.ion.ucl. ac.uk/spm) implemented in MATLAB. Scans were realigned, normalised, time-corrected, and spatially smoothed by an $8 \mathrm{~mm}$ FWHM gaussian kernel [5,19]. Low-pass and high-pass frequency filters were applied to the time series. Individual events were modelled by a standard synthetic haemodynamic response function and its temporal derivative. Bilateral trials in which the left face was perceived versus extinguished (as determined by the patient's responses during scanning) were modelled separately, as were trials with unilateral LVF faces. Movement parameters derived from the realignment correction (for all six possible directions) were also entered into the design matrix as covariates of no interest. Analysis generated statistical parametric maps of the $t$-statistic (SPM $\{t\}$ ), resulting from linear contrasts between different event conditions, which were then transformed to a normal distribution (SPM $\{Z\})$. Activations were selected only if surviving a threshold of $P=0.001$ uncorrected at the voxel level, with a significance of $P<0.05$ at the cluster level. A small volume correction (SVC) using an $8 \mathrm{~mm}$ sphere was applied to $P$-values for the amygdala where indicated, in keeping with our a priori hypothesis of amygdala involvement in the processing of fearful expressions [52]. For descriptive purposes, we also report additional foci passing an uncorrected threshold of $P<0.001$ at the voxel level when not significant at the cluster level if these were consistent with a priori predictions. 

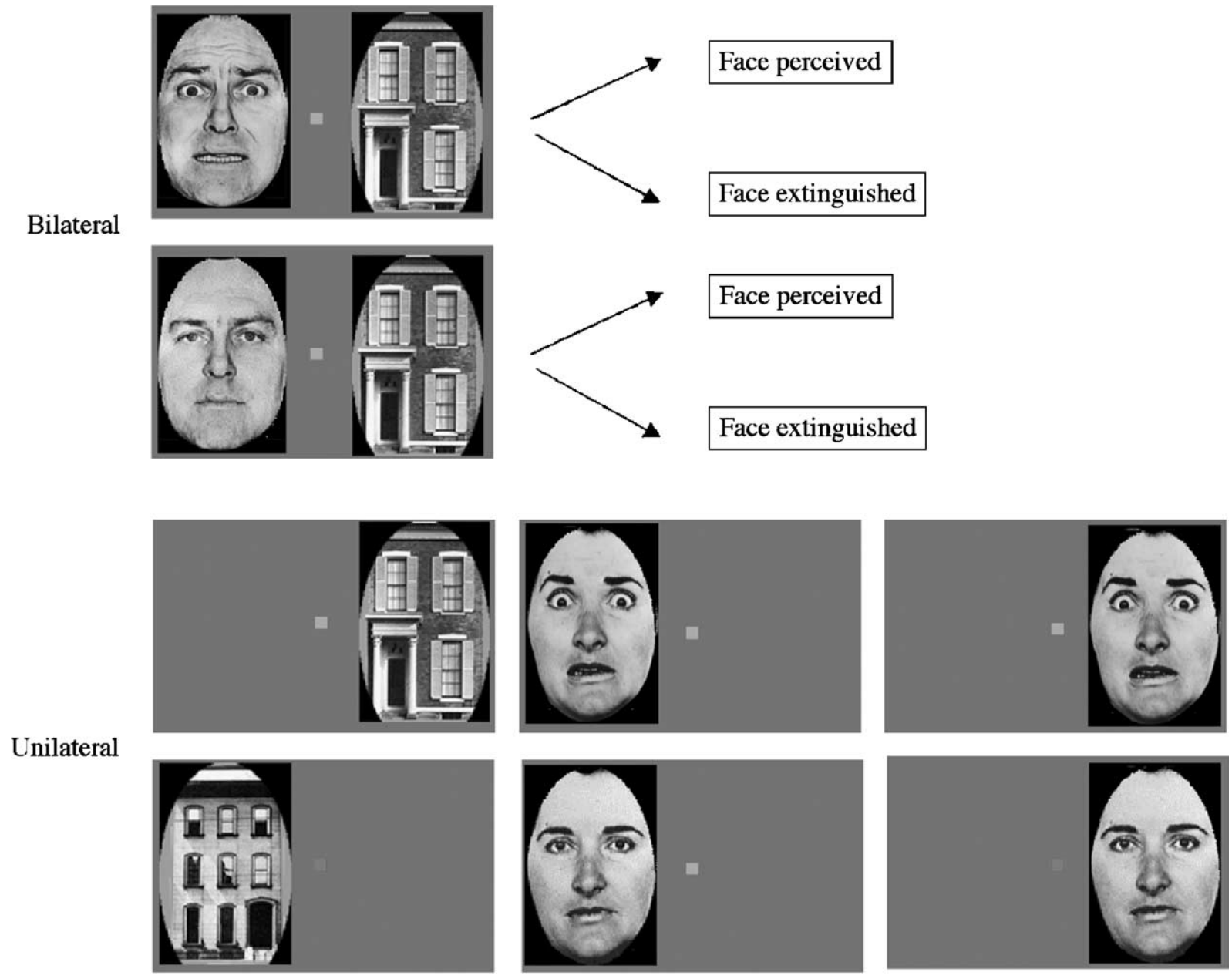

Fig. 2. Example stimuli. Critical trials were BSS with a face in the LVF and a house in the RVF, where the face was either consciously perceived or extinguished by the patient. Faces had either a fearful or neutral expression (half of trials each). A central fixation point instructed the patient either to respond by a key-press (indicating whether just a house was seen, just a face, or both stimulus types) if it was green (90\% of trials during scanning) or to withhold from responding if it was red (10\% of trials during scanning), thus ensuring accurate central fixation. Neural effects produced by extinguished (fearful or neutral) faces were examined by contrasting the critical BSS trials against unilateral trials with a right house alone. Neural effects produced by consciously seen faces were examined by comparing BSS trials with detected versus extinguished faces. The critical BSS trials allowed a factorial analysis testing the effect of emotion (fearful or neutral) and perception (extinguished or detected) for LVF faces separately, as well as any interaction between these two factors.

\section{Results}

\subsection{Behavioural performance during fMRI}

Across the three scanning sessions, the patient correctly reported $93 \%$ of unilateral faces and houses in the RVF. He missed only $37 \%$ of unilateral faces in the LVF, with no difference between faces with fearful and neutral expression, and no misidentification errors between faces and houses. On the critical bilateral trials, he missed $68 \%$ of left-side neutral faces and $63 \%$ of left-side fearful faces. This demonstrates a significant extinction pattern, with perception of LVF stimuli relatively preserved for a single stimulus, but compromised by a competing RVF stimulus $\left(\chi^{2}(1)=21.6, P<0.001\right)$. Fearful faces underwent less extinction than neutral faces on the first and second sessions (55\% versus $75 \%$ missed and
$88 \%$ versus $100 \%$, respectively, $\chi^{2}(1)=4.04, P=0.044$ ), but not on the third (46\% versus $33 \%)$. The patient correctly withheld response on all but one "no-go" trial.

\subsection{Functional imaging}

\subsubsection{Conscious perception}

We first examined neural responses evoked by visual stimuli consciously seen by the patient. Areas specifically activated by LVF stimuli were determined by comparing all unilateral left versus right trials, when the patient correctly reported the stimuli, regardless of category (i.e. faces or houses). Activation was found in striate occipital cortex and posterior lingual gyrus of the right (damaged) hemisphere, together with activations in left inferior parietal cortex and insula (Table 1). Unilateral trials were also used to determine 
Table 1

Activations produced by consciously seen stimuli

\begin{tabular}{|c|c|c|c|c|c|c|}
\hline \multirow[t]{2}{*}{ Side } & \multirow[t]{2}{*}{ Brain areas } & \multicolumn{3}{|c|}{ Coordinates } & \multirow[t]{2}{*}{$Z$-score } & \multirow[t]{2}{*}{$P$-value } \\
\hline & & $x$ & $y$ & $z$ & & \\
\hline \multicolumn{7}{|c|}{ LVF > RVF (regardless of stimulus type) } \\
\hline $\mathrm{R}$ & Lingual gyrus & 32 & -78 & -16 & 4.18 & $<0.001$ \\
\hline $\mathrm{L}$ & Posterior insula & -38 & -18 & 20 & 4.14 & $<0.001$ \\
\hline $\mathrm{R}$ & Striate cortex (posterior) & 10 & -96 & -10 & 4.04 & $<0.001$ \\
\hline $\mathrm{R}$ & Striate cortex (anterior) & 8 & -74 & 2 & 3.69 & $<0.001^{* *}$ \\
\hline $\mathrm{L}$ & Supramarginal gyrus & -56 & -38 & 22 & 3.93 & $<0.001$ \\
\hline $\mathrm{L}$ & Supramarginal gyrus & -50 & -38 & 16 & 3.62 & $<0.001$ \\
\hline \multicolumn{7}{|c|}{ Faces $>$ houses (regardless of field) } \\
\hline $\mathrm{L}$ & Lingual gyrus & -18 & -82 & -6 & 4.09 & $<0.001$ \\
\hline $\mathrm{L}$ & Inferior occipital gyrus & -12 & -92 & -20 & 3.81 & $<0.001$ \\
\hline $\mathrm{R}$ & Fusiform gyrus & 30 & -60 & -20 & 3.75 & $<0.001$ \\
\hline \multicolumn{7}{|c|}{ Fearful > neutral faces } \\
\hline $\mathrm{L}$ & Fusiform gyrus & -32 & -70 & -14 & 5.23 & $<0.001$ \\
\hline $\mathrm{L}$ & Retrosplenial cortex & -18 & -58 & 26 & 4.56 & $<0.001^{* *}$ \\
\hline \multirow[t]{2}{*}{$\mathrm{L}$} & Amygdala & -30 & 6 & -34 & 4.39 & $<0.001^{*}$ \\
\hline & & -20 & 0 & -36 & 3.34 & \\
\hline $\mathrm{R}$ & Amygdala & 24 & -6 & -34 & 3.40 & $<0.001^{* *}$ \\
\hline $\mathrm{R}$ & Middle frontal gyrus & 42 & 56 & -4 & 4.22 & $<0.001$ \\
\hline $\mathrm{L}$ & Lateral orbitofrontal & -30 & 30 & -24 & 3.93 & $<0.001^{* *}$ \\
\hline $\mathrm{L}$ & Cerebellum & -28 & -42 & -40 & 4.3 & $<0.001$ \\
\hline
\end{tabular}

All activations survived a threshold of $P<0.05$ at the cluster level, except ${ }^{*} P<0.05$ corrected for volume of interest, ${ }^{* *} P=0.001$ uncorrected Coordinates refer to normalised Talairach \& Tournoux stereotactic space, but anatomical areas correspond to the patient's own brain as defined from his MRI scan.

areas responding more to faces than to houses, regardless of side (LVF or RVF) and expression. This revealed activations in right fusiform gyrus and bilateral inferior temporal cortex (Table 1 and Fig. 3A). These results are in accordance with previous findings in normal subjects (e.g. $[23,26])$ and in this patient [43].

The effect of emotional expression, when faces were consciously seen, was assessed by a conjunction analysis [41] looking at areas commonly activated by fearful more than neutral faces across different conditions, i.e. both on unilateral trials with a RVF face and on bilateral trials with a perceived LVF face. (Unilateral LVF faces were not included in this conjunction because of an insufficient number of such events for each expression.) Increased activity due to fearful facial expression was observed in left amygdala and left fusiform gyrus (Fig. 3B), as well as in the left retrosplenial cortex, left orbitofrontal cortex, and right anterior middle frontal gyrus (Table 1). Right amygdala activation was also present but did not survive correction for multiple comparisons (Table 1). All these areas have previously been implicated in emotional face processing for normal subjects (e.g. $[10,35,37,51])$.

\subsubsection{Unconscious perception in bilateral trials}

We next examined the neural response evoked by extinguished faces (regardless of expression). Bilateral trials with an unseen face in the LVF and a house in the RVF (BSS extinguished) were compared to unilateral trials with a RVF house alone (i.e. events associated with same awareness and response, but different contralesional stimuli). This revealed significant activations in bilateral occipital areas, including peaks in right primary striate cortex and bilateral cunei (Table 2). These results confirm other fMRI data on extinction $[43,47]$ showing activation of right posterior visual cortex by unseen LVF stimuli. ${ }^{1}$

\subsubsection{Conscious and unconscious perception of emotional faces in bilateral trials}

A major question in our study concerned the neural response to emotional stimuli when extinguished from awareness. The critical BSS trials constituted a $2 \times 2$ factorial analysis allowing us to determine main effects of emotion (fearful versus neutral expression) and awareness (consciously seen versus extinguished faces) separately, as well as any significant modulation of emotional responses as a function of awareness (interaction term).

The main effect of fear (BSS with seen + extinguished fearful faces $>$ BSS with seen + extinguished neutral faces) identified brain regions responding to emotional expressions irrespective of whether the face was consciously seen or not. In keeping with the findings above, such increases were

\footnotetext{
${ }^{1}$ We also examined areas with increased activity for the opposite contrast of extinguished versus perceived stimuli in LVF (regardless of category). This revealed selective activation of cuneus $(x=-8, y=-66$, $z=26, Z=4.58)$ and peristriate cortex $(x=-14, y=-58, z=14$, $Z=3.84$ ) in left medial occipital lobe. Such increases in the intact hemisphere on extinction trials might reflect the neural effects of a competition between bilateral perceived stimuli across hemifields [18].
} 

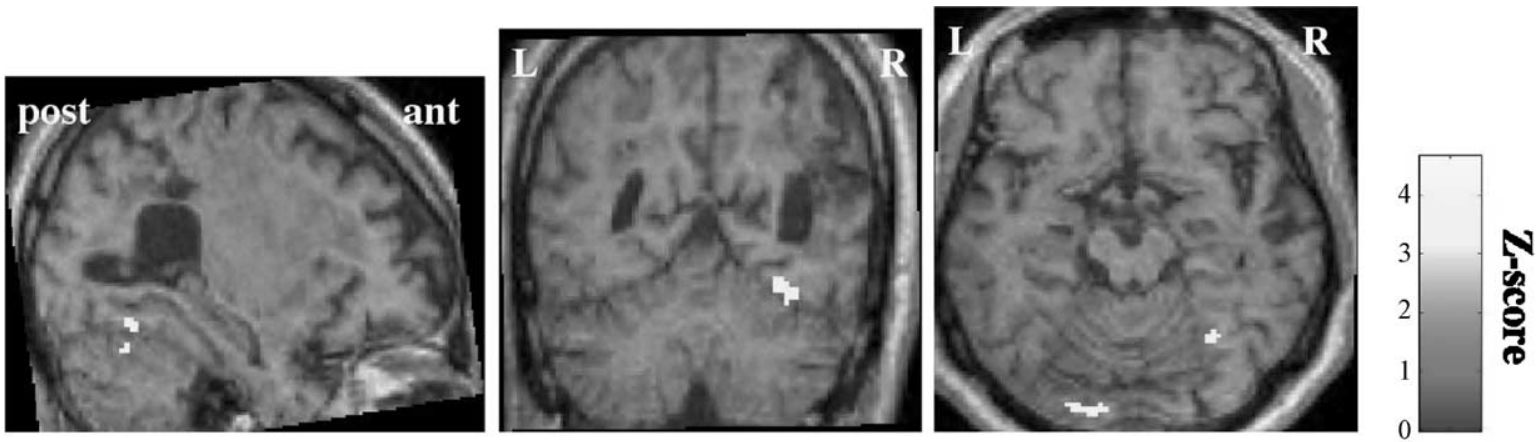

(A)
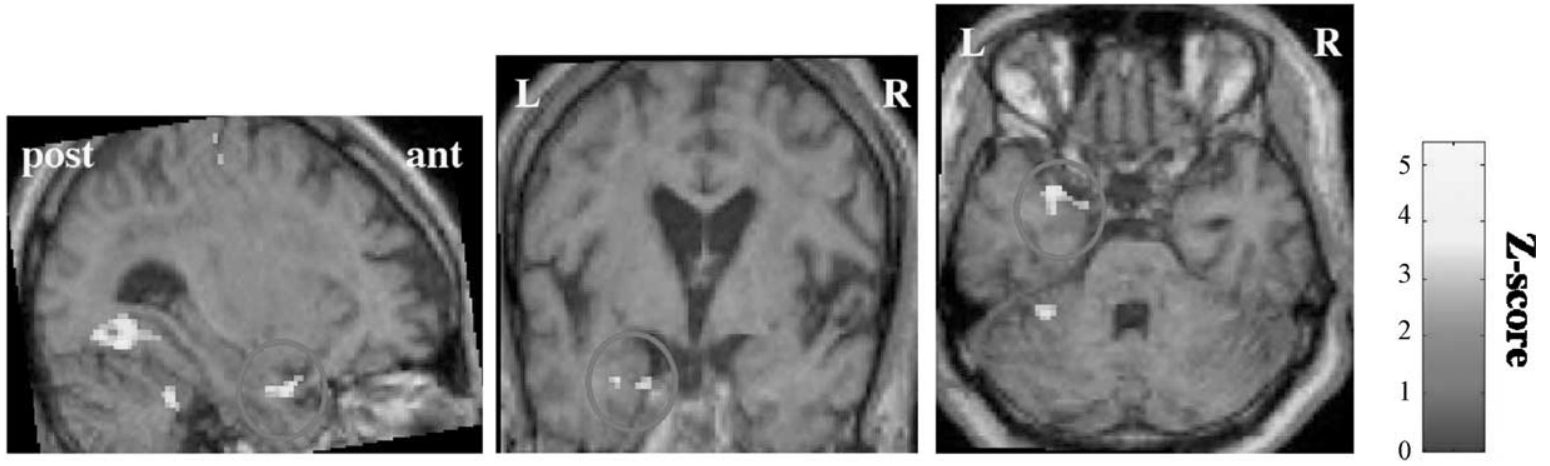

(B)

Fig. 3. Peaks of activation shown superimposed on the structural MRI brain scan of the patient. (A) Activity specifically evoked by consciously seen faces, as opposed to seen houses, was observed in the right fusiform cortex $(x=30, y=-60, z=-20)$, irrespective of emotional expression and field of presentation. (B) Activity specifically evoked by faces with a fearful expression, as opposed to neutral expression, was observed in the left amygdala, with two peaks merging anteriorly $(x=-30, y=6, z=-34$ and $x=-20, y=0, z=-36$, encircled in red), as well as in the left fusiform cortex ( $x=-32$, $y=-70, z=-14)$ and left lateral orbitofrontal cortex $(x=-30, y=30, z=-24$; not shown here), irrespective of field of presentation. Activity in the fusiform cortex, but not in left amygdala and orbitofrontal cortex, was modulated by awareness of the faces, as opposed to extinction (see Fig. 4).

Table 2

Activations produced by extinguished faces in LVF

\begin{tabular}{|c|c|c|c|c|c|c|}
\hline \multirow[t]{2}{*}{ Side } & \multirow[t]{2}{*}{ Brain areas } & \multicolumn{3}{|c|}{ Coordinates } & \multirow[t]{2}{*}{$Z$-score } & \multirow[t]{2}{*}{$P$-value } \\
\hline & & $x$ & $y$ & $z$ & & \\
\hline \multicolumn{7}{|c|}{ All extinguished faces $>$ RVF house alone } \\
\hline $\mathrm{L}$ & Posterior lingual gyrus & -8 & -74 & 2 & 3.82 & $<0.001^{* *}$ \\
\hline $\mathrm{L}$ & Pulvinar & -12 & -28 & 4 & 3.8 & $<0.001^{* *}$ \\
\hline $\mathrm{L}$ & Fusiform gyrus & -18 & -50 & -22 & 3.76 & $<0.001^{* *}$ \\
\hline $\mathrm{L}$ & Cuneus & -8 & -70 & 32 & 3.39 & $<0.001$ \\
\hline $\mathrm{R}$ & Cuneus & 10 & -92 & 18 & 3.26 & $0.001^{* *}$ \\
\hline $\mathrm{R}$ & Striate cortex & 0 & -86 & 2 & 3.26 & $0.001^{* *}$ \\
\hline $\mathrm{L}$ & Inferior temporal gyrus & -46 & -26 & -22 & 3.28 & $0.001^{* *}$ \\
\hline \multicolumn{7}{|c|}{ Only extinguished fearful faces $>$ RVF house alone } \\
\hline $\mathrm{L}$ & Amygdala & -26 & 0 & -28 & 3.37 & $<0.001^{*}$ \\
\hline $\mathrm{R}$ & Posterior lingual gyrus & 10 & -76 & 0 & 4.15 & $<0.001$ \\
\hline
\end{tabular}

Only extinguished neutral faces > RVF alone

No additional foci

All activations survived a threshold of $P<0.05$ at the cluster level, except ${ }^{*} P<0.05$ corrected for volume of interest, ${ }^{* *} P=0.001$ uncorrected. Coordinates refer to normalised Talairach \& Tournoux stereotactic space, but anatomical areas correspond to the patient's own brain as defined from his MRI scan. 
Table 3

Activations produced by critical BSS trials

\begin{tabular}{|c|c|c|c|c|c|c|}
\hline \multirow[t]{2}{*}{ Side } & \multirow[t]{2}{*}{ Brain areas } & \multicolumn{3}{|c|}{ Coordinates } & \multirow[t]{2}{*}{ Z-score } & \multirow[t]{2}{*}{$P$-value } \\
\hline & & $x$ & $y$ & $z$ & & \\
\hline \multicolumn{7}{|c|}{ Fearful > neutral expression of left faces in BSS (regardless of awareness) } \\
\hline $\mathrm{R}$ & Intraparietal sulcus & 30 & -66 & 40 & 4.26 & $<0.001$ \\
\hline $\mathrm{L}$ & Fusiform gyrus & -32 & -60 & -12 & 3.73 & $<0.001$ \\
\hline $\mathrm{L}$ & Amygdala & -30 & 6 & -34 & 3.46 & $<0.001^{*}$ \\
\hline $\mathrm{L}$ & Lateral orbitofrontal & -30 & 28 & -22 & 3.41 & $<0.001^{* *}$ \\
\hline \multicolumn{7}{|c|}{ Conscious awareness $>$ extinction of left faces in BSS (regardless of expression) } \\
\hline $\mathrm{L}$ & Inferior parietal cortex & -58 & -34 & 42 & 4.28 & $<0.001$ \\
\hline $\mathrm{L}$ & Fusiform gyrus & -38 & -64 & -20 & 4.21 & $<0.001$ \\
\hline $\mathrm{L}$ & Middle frontal gyrus & -38 & 58 & -6 & 4.05 & $<0.001^{* *}$ \\
\hline $\mathrm{L}$ & Intraparietal sulcus & -34 & -50 & 42 & 3.85 & $<0.001$ \\
\hline $\mathrm{L}$ & Middle frontal gyrus & -36 & 38 & 0 & 3.6 & $<0.001^{* *}$ \\
\hline \multicolumn{7}{|c|}{ Increased response to left fearful faces in BSS only with conscious awareness } \\
\hline $\mathrm{R}$ & Anterior parietal cortex & 56 & -22 & 32 & 4.86 & $<0.001$ \\
\hline $\mathrm{L}$ & Anterior parietal cortex & -40 & -22 & 40 & 4.84 & $<0.001$ \\
\hline $\mathrm{R}$ & Temporal pole & 50 & 8 & -48 & 4.27 & $<0.001$ \\
\hline $\mathrm{R}$ & Ventrolateral frontal & 28 & 42 & -16 & 4.21 & $<0.001$ \\
\hline $\mathrm{L}$ & Fusiform gyrus & -30 & -64 & -16 & 4.15 & $<0.001$ \\
\hline $\mathrm{L}$ & Medial frontal gyrus & -2 & 52 & 34 & 4.01 & $<0.001$ \\
\hline
\end{tabular}

All activations survived a threshold of $P<0.05$ at the cluster level, except ${ }^{*} P<0.05$ corrected for volume of interest, ${ }^{* *} P=0.001$ uncorrected. Coordinates refer to normalised Talairach \& Tournoux stereotactic space, but anatomical areas correspond to the patient's own brain as defined from his MRI scan.

found in left amygdala (Table 3), together with left fusiform cortex, left orbitofrontal cortex, and right intraparietal sulcus (Table 3). All these regions responded to fearful more than neutral expressions regardless of awareness.

The main effect of awareness (BSS with seen fearful + seen neutral faces $>$ BSS with extinguished fearful + extinguished neutral faces) tested for regions specifically activated by conscious perception of faces in the LVF (as opposed to extinction), irrespective of emotional expression, for physically equivalent displays. This revealed significant increases in several areas of the left-hemisphere (Table 3), including left fusiform, left parietal, and left frontal cortex. ${ }^{2}$

Finally, the most direct test for determining the extent to which the response to fearful faces was modulated by awareness of the faces is to identify brain regions showing an interaction between emotion and awareness on BSS trials, responding more to fear when faces were perceived versus extinguished ([BSS with seen fearful faces $>$ seen neutral faces]-[BSS with extinguished fearful faces $>$ extinguished neutral faces]). Significant increases were found in left fusiform gyrus, plus bilateral anterior parietal regions, right

\footnotetext{
${ }^{2}$ For completeness, we also examined the effect of conscious perception versus extinction of unilateral LVF stimuli, regardless of category (although there were relatively few missed trials). This contrast revealed increases in bilateral extrastriate visual cortex (right fusiform: $x=20$, $y=-62, z=12, Z=3.97$; right middle temporal gyrus: $x=58$, $y=-26, z=-8, Z=3.91$; left lingual gyrus: $x=-20, y=-92$, $z=-12, Z=4.22)$, as well as in left inferior frontal cortex $(x=-60$, $y=-24, z=12, Z=3.87 ; x=-46, y=-2, z=12, Z=3.82$ ), left parietal cortex $(x=-12, y=-68, z=40, Z=3.59)$, and mid cingulate gyrus ( $x=0, y=-28, z=54, Z=3.77)$.
}

anterior temporal pole, and bilateral frontal cortex (Table 3 and Fig. 4). Notably, this interaction was not significant in the left amygdala even at a liberal statistical threshold ( $Z=1.58, P=0.057$ uncorrected, $P=0.64$ corrected).

\subsubsection{Comparison of fearful and neutral faces in extinguished bilateral trials}

An additional analysis to confirm emotional responses without conscious perception (e.g. in amygdala) examined BSS trials in which either fearful or neutral faces were extinguished in the LVF, compared with RVF houses alone. This showed that extinguished fearful faces produced significant activation of left amygdala $(Z=3.41, P<0.05$ corrected for small volume) in addition to activating other striate and extrastriate visual areas (Table 2). By contrast, unseen neutral faces in extinguished BSS versus unilateral RVF trials did not produce a reliable effect in the left amygdala $(Z=$ 1.98, $P=0.024$ uncorrected, $P=0.58$ corrected).

Direct comparison of all extinguished faces revealed that unseen fearful as opposed to unseen neutral faces increased activity in several areas including bilateral lingual gyri $(x=$ $12, y=-76, z=-2, Z=4.52$; and $x=18, y=-60$, $z=-6, Z=4.07)$, bilateral orbitofrontal cortex $(x=4$, $y=50, z=-20, Z=4.35$; and $x=-18, y=32$, $z=-14, Z=4.05)$, left pulvinar $(x=18, y=-32$, $z=2, Z=4.29$ ), and right superior intraparietal sulcus $(x=36, y=-74, z=44, Z=4.40$; all $P<0.001$ uncorrected, $P<0.05$ at the cluster level). This contrast did not reach significance in the amygdala $(Z=1.37, P=$ 0.086 uncorrected, $P=0.75$ corrected), but the lack of a significant amygdala effect for this comparison can be 
(A) R. fusiform

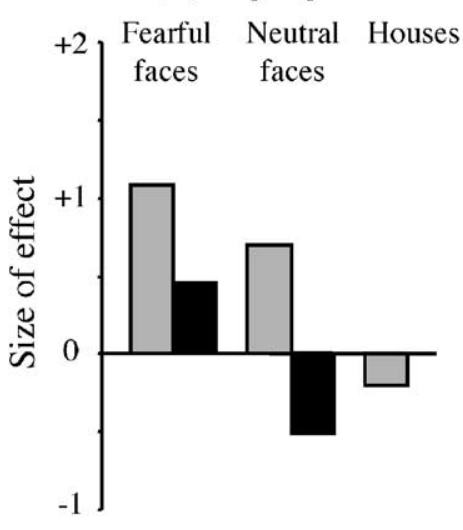

(C) L. amygdala

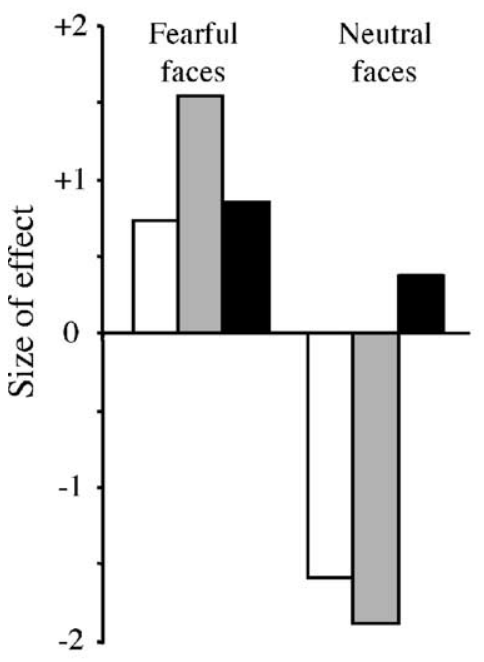

(B) L. fusiform

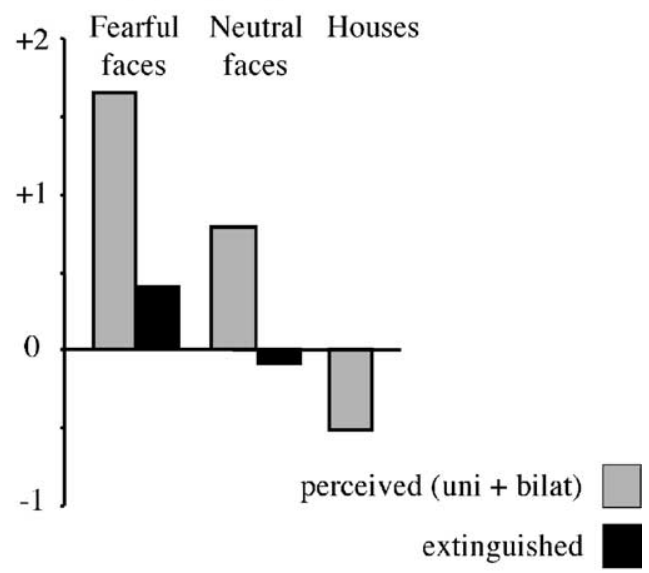

(D) L. orbitofrontal

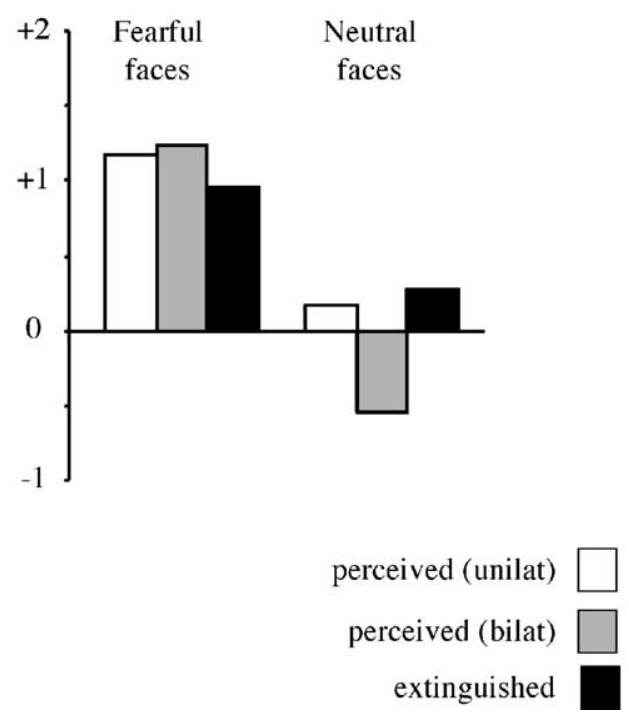

Fig. 4. Size of activation for activated brain areas shown in Fig. 3 (peak voxels weighted by smoothing of their surround), averaged across conditions and scanning sessions (arbitrary units). (A, B) Activity in both right and left fusiform cortex increased when faces were consciously perceived, as opposed to extinguished, irrespective of emotional expression. In addition, bilateral fusiform areas showed an enhanced response to fearful as compared to neutral expression, both when the fares were perceived or extinguished. (C) Activity in left amygdala increased in response to fearful faces both when consciously perceived and extinguished. The left amygdala also showed a weak response to neutral faces when these were extinguished, but no response to neutral faces when these were consciously seen (irrespective of field of presentation, in unilateral right or bilateral trials). (D) The lateral orbitofrontal cortex also responded to fearful faces during both conscious perception and extinction.

explained by a closer inspection of amygdala activity across conditions. As Fig. 4 shows, the left amygdala response to neutral faces was higher when the faces were extinguished than when consciously perceived. This unexpected increase in activity for neutral faces with extinction, versus conscious perception, was significant $(Z=3.61, P<0.05$ corrected for small volume).

\section{Discussion}

In this right-parietal patient with left neglect and extinction, consciously perceived faces with a fearful expression activated bilateral visual areas in fusiform gyri and adjacent temporo-occipital cortex, as well as the left amygdala and other emotion-related regions in mediofrontal and orbitofrontal cortex. This pattern is consistent with findings in neurologically intact subjects $[10,23,26,35,37,46,51]$. Our novel results demonstrate that several areas responded to the emotional significance of faces irrespective of awareness. In particular, the left amygdala was activated by fearful faces in the contralesional LVF both when seen and when extinguished. Moreover, we found no interaction between emotion and awareness in the amygdala. In our factorial analysis, the left amygdala exhibited a main effect of fearful expression without any additional effect due to awareness of 
the faces (see Table 3), suggesting that its response to fear was not significantly modulated as a function of conscious perception versus extinction.

Other areas also responded to fearful more than neutral faces regardless of awareness, including left fusiform, lateral orbitofrontal, and right intraparietal cortex (superior to the lesion). Our novel findings for orbitofrontal cortex appears consistent with a recent report of unconscious responses to subliminal stimuli in orbitofrontal cortex, as revealed by intracerebral event-related potentials in human subjects [9]. By contrast, left fusiform cortex was more activated by consciously perceived versus extinguished faces, irrespective of expression. This accords with previous evidence that fusiform activity correlates with awareness of faces in normal subjects $[20,45]$.

Our results provide a possible neural substrate for recent behavioural findings in patients with spatial neglect and extinction [48,49] suggesting that emotional properties of contralesional stimuli can be extracted despite pathological inattention and unawareness. Our findings show that processing of extinguished stimuli can proceed well beyond early visual pathways. This extends previous neuropsychological observations of residual processing based on visual grouping [13] or object recognition mechanisms [8], thought to depend on occipito-temporal areas. It also extends recent fMRI data showing activation of primary visual cortex and posterior temporal regions by extinguished stimuli $[14,43,47]$. Here, extinguished fearful faces activated not only extrastriate visual cortex, but also left amygdala, in anterior and medial regions of the temporal lobe, as well as orbitofrontal cortex and superior parietal cortex, all structurally spared by the lesion in GK. This suggests that extinguished emotional stimuli can receive substantial processing in distributed brain areas, quite distant from early sensory cortex, yet still not reach awareness.

\subsection{Emotional responses without awareness}

These results support proposals that the amygdala can process fear-related information with a large degree of automaticity, e.g. without conscious perception or selective attention [4,31]. Other PET and fMRI studies in normal subjects demonstrated amygdala responses to fear-conditioned stimuli $[11,29]$ and fearful faces [10,35,37,51], even when explicit perception of the faces was suppressed in healthy subjects by masking [36,51] or inattention [46]; or when faces were presented in the scotoma of a patient with blindsight [34]. In our patient, the left amygdala was still activated when he extinguished fearful faces in LVF and reported only the presence of a RVF house, indicating that the amygdala may detect emotionally-relevant stimuli despite attention being engaged by another competing stimulus at a separate location. Taken together, these results suggest that emotional inputs to the amygdala may be at least partly independent of processing in occipital and fusiform cortex associated with visual awareness.
Previous anatomical and imaging data have pointed to a subcortical route (via superior colliculus and pulvinar) for rapid and unconscious inputs to the amygdala, bypassing primary visual cortex [31,34]. In GK, extinguished faces increased activity of left pulvinar, more when faces were fearful than neutral. However, extinguished faces in the LVF (as compared to RVF houses alone) also activated right primary visual cortex and bilateral extrastriate areas (see Table 2). Inputs from extinguished stimuli engaging either the pulvinar or visual occipital cortex might still project to fusiform areas [12] at a level below threshold for conscious perception of a face, but sufficient to trigger an amygdala response.

Face-selective fusiform areas showed an enhanced response to fearful as compared to neutral expression, both when the faces were perceived or extinguished (see Fig. 3). This was additive to an increase associated with awareness versus extinction. Such emotional enhancement could reflect feedback influences from amygdala on fusiform cortex, independent of awareness and attention $[1,35,46]$. This would converge with recent results from single-cell recording in temporal cortex of the monkey [44] and functional imaging in healthy humans $[35,46]$.

The lateral orbitofrontal cortex was also activated by extinguished fearful faces, and receives direct inputs from the amygdala [1]. Orbitofrontal cortex has been implicated in emotional processing across a variety of conditions (e.g. $[39,46])$. Moreover, activity in both orbitofrontal and parietal areas has recently been associated with faster orienting of spatial attention towards emotional stimuli [3], as found behaviourally in healthy subjects [33] and in some parietal patients despite hemispatial neglect $[48,49]$. An enhanced processing of emotional stimuli in fusiform and orbitofrontal areas might underlie the greater salience of such stimuli for perceptual awareness. Here, however, GK detected fearful better than neutral faces only during the first and second scan sessions, not the third. It is possible that emotional effects on attention and awareness are liable to habituation [10,11], especially after repeated exposure to the same stimuli, as here.

\subsection{Differences in neural responses with and without awareness}

An unexpected finding was that neutral faces activated the amygdala more when extinguished than when consciously perceived (see Fig. 4). We speculate that a neutral face represents more potential threat when outside the current focus of attention, consistent with a more general function for the amygdala processing of salient stimuli with social and emotional meaning, beyond just fear recognition and conditioning [32,50]. Increased amygdala activity has also been found for neutral faces of strangers versus familiar people [15], social out-group versus in-group [22,40], or direct eye-contact versus averted gaze $[21,27]$. Moreover, several studies found that masked stimuli (including angry faces and aversive pictures) can elicit stronger emotional behavioural reactions and greater amygdala activation than the same stimuli when 
unmasked $[2,28,33]$. This may accord with the view that while crude emotional processing can take place rapidly and unconsciously, the engagement of conscious cognitive processes can modify or suppress these evaluations [38].

Finally, the present study corroborates and extends other recent fMRI data on visual extinction after parietal damage. These results demonstrate that contralesional stimuli can activate intact visual cortex in the damaged hemisphere, despite unawareness $[43,47]$. On the other hand, the same contralesional stimuli can produce greater activation in several areas of the left-hemisphere when consciously perceived, including fusiform, posterior parietal, and inferior frontal cortex. Differential eye-position cannot be entirely ruled out and might in principle contribute, though note that a colour monitoring task was performed at central fixation to minimise this. Moreover, even if unintended rightward saccades were more frequent on extinguished trials, this would not undermine our most critical findings of a differential effect for extinguished LVF emotional stimuli. The pattern of left-hemisphere activation associated with conscious versus unconscious perception of contralesional faces was remarkably similar in this study and a previous investigation [47], with a different patient and different stimuli. Although some of these left-hemisphere activations (e.g. inferior frontal cortex) could in principle reflect covert verbal processes (not strictly required by the task), rather than visual awareness per se, this seems unlikely to explain all these activations, given the specific anatomical loci observed. Moreover, it is increasingly thought that visual awareness may reflect the availability of information to a diffuse network (including but not restricted to availability for verbal report) (e.g. [6]), rather than merely activation within visual cortex as traditionally conceived.

\section{Acknowledgements}

We thank patient GK for his participation, and Emiliano Macaluso for technical help during scanning. Supported by grants the Wellcome Trust (JD, MH, RJD), Human Frontier Science Projects (JD), and the Swiss National Science Foundation (PV). JD holds a Royal Society-Wolfson Research Merit Award. This research was facilitated by an MRC co-operative awards.

\section{References}

[1] Aggleton JP, Burton MJ, Passingham RE. Cortical and subcortical afferents to the amygdala of the rhesus monkey (Macaca mulatta). Brain Research 1980;190:321-32.

[2] Armony JL, Dolan RJ. The neural substrates of emotion-attention interactions in humans. Society of Neuroscience Abstracts, 2000

[3] Armony JL, Dolan RJ. Modulation of spatial attention by fearconditioned stimuli: an event-related fMRI study. Neuropsychologia 2002;40:817-26.

[4] Armony JL, LeDoux JE. How danger is encoded: towards a systems, cellular, and computational understanding of cognitive-emotional interactions in fear. In: Gazzaniga MS, editor. The new cognitive neurosciences. Cambridge, MA: MIT Press, 2000. p. 1067-80.

[5] Ashburner J, Friston K. Multimodal image coregistration and partitioning - a unified framework. Neuroimage 1997;6:209-17.

[6] Baars BJ. The conscious access hypothesis: origins and recent evidence. Trends in Cognitive Science 2002;6:47-52.

[7] Bender MB, Teuber HL. Phenomena of fluctuation, extinction, and completion in visual perception. Archives of Neurology and Psychiatry 1946;55:627-58.

[8] Berti A, Rizzolatti G. Visual processing without awareness: evidence from unilateral neglect. Journal of Cognitive Neuroscience 1992;4:345-51.

[9] Brazdil M, Rektor I, Daniel P, Dufek M, Jurak P. Intracerebral event-related potentials to subthreshold target stimuli. Clinical Neurophysiological 2001;112:650-61.

[10] Breiter HC, Etcoff NL, Whalen PJ, Kennedy WA, Rauch SL, Buckner $\mathrm{RL}$, et al. Response and habituation of the human amygdala during visual processing of facial expression. Neuron 1996;17:875-87.

[11] Buchel C, Morris J, Dolan RJ, Friston KJ. Brain systems mediating aversive conditioning: an event-related fMRI study. Neuron 1998;20:947-57.

[12] Clarke S, Riahi-Arya S, Tardif E, Cottier Eskenasy AC, Probst A. Thalamic projections of the fusiform gyrus in man. European Journal of Neuroscience 1999;11:1835-8.

[13] Driver J, Vuilleumier P. Perceptual awareness and its loss in unilateral neglect and extinction. Cognition 2001;79:39-88.

[14] Driver J, Vuilleumier P, Eimer M, Rees G. Functional MRI and evoked potential correlates of conscious and unconscious vision in parietal extinction patients. Neuroimage 2001;14:68-75.

[15] Dubois S, Rossion B, Schiltz C, Bodart JM, Michel C, Bruyer R, et al. Effect of familiarity on the processing of human faces. Neuroimage 1999;9:278-89.

[16] Ekman P, Friesen W. Pictures of facial affect. Palo Alto: Consulting Psychologists Press, 1976.

[17] Esteves F, Dimberg U, Öhman A. Automatically elicited fear: conditioned skin conductance responses to masked facial expressions. Cognition and Emotion 1994;9:99-108.

[18] Fink GR, Driver J, Rorden C, Baldeweg T, Dolan RJ. Neural consequences of competing stimuli in both visual hemifields: a physiological basis for visual extinction. Annals in Neurology 2000;47:440-6.

[19] Friston KJ, Holmes AP, Worsley KJ, Poline JB, Frith CD, Frackowiak RS. Statistical parametric maps in functional imaging: a general linear approach. Human Brain Mapping 1995;2:189-210.

[20] George N, Dolan RJ, Fink GR, Baylis GC, Russell C, Driver J. Contrast polarity and face recognition in the human fusiform gyrus. Nature Neuroscience 1999;2:574-80.

[21] George N, Driver J, Dolan RJ. Seen gaze-direction modulates fusiform activity and its coupling with other brain areas during face processing. Neuroimage 2001;13:1102-12.

[22] Hart AJ, Whalen PJ, Shin LM, McInerney SC, Fischer H, Rauch SL. Differential response in the human amygdala to racial out-group versus ingroup face stimuli. Neuroreport 2000;11:2351-5.

[23] Haxby JV, Hoffman EA, Gobbini MI. The distributed human neural system for face perception. Trends in Cognitive Neuroscience 2000;4:223-32.

[24] Heilman KM, Watson RT, Valenstein E. Neglect and related disorders. In: Heilman KM, Valenstein E, editors. Clinical neuropsychology, 3rd ed. New York: Oxford University Press, 1993. p. 279-336.

[25] Husain M, Mannan S, Hodgson T, Woljciulik E, Driver J, Kennard C. Impaired spatial working memory across saccades contributes to abnormal search in parietal patients. Brain 2001;124:941-52.

[26] Kanwisher N, McDermott J, Chun MM. The fusiform face area: a module in human extrastriate cortex specialized for face perception. Journal of Neuroscience 1997;17:4302-11.

[27] Kawashima R, Sugiura M, Kato T, Nakamura A, Hatano K, Ito K, et al. The human amygdala plays an important role in gaze monitoring: a PET study. Brain 1999;122:779-83. 
[28] Kubota Y, Sato W, Murai T, Toichi M, Ikeda A, Sengoku A. Emotional cognition without awareness after unilateral temporal lobectomy in humans. Journal of Neuroscience 2000;20(RC97):1-5.

[29] LaBar KS, Gatenby JC, Gore JC, LeDoux JE, Phelps EA. Human amygdala activation during conditioned fear acquisition and extinction: a mixed-trial fMRI study. Neuron 1998;20:947-57.

[30] Lang PJ, Bradley MM, Cuthbert BN. Emotion and attention: stop, look, and listen. Current Psychology of Cognition (Cahiers de Psychologie Cognitive) 1998;17:997-1020.

[31] LeDoux JE. The emotional brain. New York: Simon \& Schuster, 1996.

[32] Leonard CM, Rolls ET, Wilson FA, Baylis GC. Neurons in the amygdala of the monkey with responses selective for faces. Behavioural Brain Research 1985;15:159-76.

[33] Mogg K, Bradley BP. Orienting of attention to threatening facial expressions presented under conditions of restricted awareness. Cognition and Emotion 1999;13:713-40.

[34] Morris J, DeGelder B, Weiskrantz L, Dolan RJ. Differential extrageniculostriate and amygdala responses to presentation of emotional faces in a cortically blind field. Brain 2001;124:1241-52.

[35] Morris J, Friston KJ, Buchel C, Frith CD, Young AW, Calder AJ, et al. A neuromodulatory role for the human amygdala in processing emotional facial expressions. Brain 1998;121:47-57.

[36] Morris J, Öhman A, Dolan RJ. Conscious and unconscious emotional learning in the human amygdala. Nature 1998;393:467-70.

[37] Morris JS, Frith CD, Perrett DI, Rowland D, Young AW, Calder AJ, et al. A differential neural response in the human amygdala to fearful and happy facial expressions. Nature 1996;383: 812-5.

[38] Murphy ST, Zajonc RB. Affect, cognition, and awareness: affective priming with optimal and suboptimal stimulus exposures. Journal of Personality and Social Psychology 1993;64:723-39.

[39] Paradiso S, Johnson DL, Andreasen NC, O'Leary DS, Watkins GL, Ponto LL, et al. Cerebral blood flow changes associated with attribution of emotional valence to pleasant, unpleasant, and neutral visual stimuli in a PET study of normal subjects. American Journal of Psychiatry 1999;156:1618-29.

[40] Phelps EA, O'Connor KJ, Cunningham WA, Funayama ES, Gatenby JC, Gore JC, et al. Performance on indirect measures of race evaluation predicts amygdala activation. Journal in Cognitive Neuroscience 2000;12:729-38.

[41] Price CJ, Moore CJ, Friston KJ. Subtractions, conjunctions, and interactions in experimental design of activation studies. Human Brain Mapping 1997;5:264-72.

[42] Rafal RD. Neglect. Current Opinion in Neurobiology 1994;4:231-6.

[43] Rees G, Wojciulik E, Clarke K, Husain M, Frith CD, Driver J. Unconscious activation of visual cortex in the damaged right-hemisphere of a parietal patient with extinction. Brain 2000;123:1624-33.

[44] Sugase Y, Yamane S, Ueno S, Kawano K. Global and fine information coded by single neurons in the temporal visual cortex. Nature 1999;400:869-73.

[45] Tong F, Nakayama K, Vaughan JT, Kanwisher N. Binocular rivalry and visual awareness in human extrastriate cortex. Neuron 1998;21: 753-9.

[46] Vuilleumier P, Armony JL, Driver J, Dolan RJ. Effects of attention and emotion on face processing in the human brain: An event-related fMRI study. Neuron 2001;3:829-41.

[47] Vuilleumier P, Sagiv N, Hazeltine E, Poldrack R, Swick D, Rafal $\mathrm{R}$, et al. Neural fate of seen and unseen faces in unilateral spatial neglect: a combined event-related fMRI and ERP study of visual extinction. Proceedings of the National Academy of Sciences of the United States of America 2001;98:3495-500.

[48] Vuilleumier P, Schwartz I. Emotional facial expressions capture attention. Neurology 2001;56:153-8.

[49] Vuilleumier P, Schwartz S. Beware and be aware: capture of attention by fear-relevant stimuli in patients with unilateral neglect. Neuroreport 2001;12:1119-22.

[50] Whalen PJ. Fear, vigilance, and ambiguity: initial neuroimaging studies of the human amygdala. Current Directions in Psychological Science 1998;7:177-88.

[51] Whalen PJ, Rauch SL, Etcoff NL, McInerney SC, Lee MB, Jenike MA. Masked presentations of emotional facial expressions modulate amygdala activity without explicit knowledge. Journal of Neuroscience 1998;18:411-8.

[52] Worsley KJ, Marrett S, Neelin P, Vandal AC, Friston KJ, Evans AC. A unified statistical approach for determining significant signals in images of cerebral activation. Human Brain Mapping 1996;4:58-73. 